\title{
Comparing computerized tomography indices and liver biopsy in liver transplantation donors for hepatosteatosis
}

\author{
Genco Gencdal ${ }^{1}$, Utku Alkara ${ }^{2}$, Murat Akyildiz ${ }^{3}$, \\ ${ }^{1}$ Department of Gastroenterology, Organ Transplantation Center, Atasehir Memorial Hospital, Istanbul, Turkey \\ ${ }^{2}$ Department of Radiology, Organ Transplantation Center, Yeni Yüzyil University School of Medicine, Istanbul, Turkey \\ ${ }^{3}$ Department of Gastroenterology, Organ Transplantation Center, Koc University School of Medicine, Istanbul, Turkey
}

Received: 2020-06-16.

Accepted: 2020-08-02

This work is licensed under a Creative Commons Attribution 4.0 International License
J Clin Med Kaz 2020; 5(59):42-45

Corresponding author:

Genco Gençdal.

E-mail: gencogencdal@yahoo.co.uk;

ORCID: https://orcid.org/0000-0002-5856-5384

\section{Abstract}

Background: Selecting the right donor to achieve a successful liver transplantation is very important; thus computerized tomography and liver biopsy are frequently applied in the diagnosis of steatosis in donors.

Purpose: We sought to investigate the efficacy of computerized tomography in the evaluation of hepatosteatosis in living donor liver transplantation donors.

Material and methods: The living donor liver transplantation cases between January 2015 and December 2017 were screened retrospectively. The study participants were divided into three groups according to the degree of steatosis determined during LB as follows: grade 0: less than 5\%, grade 1: $6 \%$ to $20 \%$, and grade 2: greater than $20 \%$. Using computerized tomography scans, hepatic attenuation value (CTL), hepatic attenuation value and spleen attenuation value ratio (CTL/S), and the difference between hepatic attenuation value and splenic attenuation values (CTL-S) were determined and the correlations of these indices and the findings of LB were compared.

Results: Of the 60 patients (42 males, mean age: $32.4 \pm 7.7$ years), 43 had grade 0,15 had grade 1, and two had grade 2 hepatosteatosis, respectively. The CTL, CTL/S, and CTL-S cutoff values were 48.3, 1.06, and 3.2 , respectively, while the sensitivity and specificity results of these cutoff values were $64.7 \%$ and $88.3 \%, 64.7 \%$ and $86 \%$, and $64.7 \%$ and $86 \%$ and the area under the curve values were determined to be $0.81,0.79$, and 0.80 ( $p<0.001)$.

Conclusion: The use of these noninvasive indices can reduce the need for liver biopsy, which is an invasive procedure, as well as lessen the associated complication and cost rates. Future prospective studies are needed on this subject.

Keywords: liver transplantation, donor, liver biopsy, computerized tomography

\section{Introduction}

Liver biopsy (LB) is the gold-standard method used currently in the diagnosis and treatment of liver diseases. Liver transplantation (Ltx) is the most effective treatment for liver cirrhosis. Living donor liver transplantation (LDLT) is usually performed in our country. Selecting the right donor to achieve a successful Ltx procedure is very important; thus, LB is often applied in the evaluation of potential liver donors. The presence of macrovesicular steatosis in the liver donor increases the risk of complications such as primary nonfunction and early graft dysfunction after LDLT. For this reason, many liver transplant centers are eliminating donors with severe hepatosteatosis. Separately, LB has some disadvantages including its invasive nature and complications like bleeding that may rarely result in death and it represents only a small sample area. For these reasons, the search for noninvasive methods that can be used in the diagnosis of hepatosteatosis continues. Computerized tomography (CT) is one of these methods and is used effectively in donor evaluation [1-4]. In this study, we aimed to investigate the efficacy of $\mathrm{CT}$ in the evaluation of hepatosteatosis in LDLT donors at our transplantation center. 


\section{Material and methods}

In this study, the files of patients who underwent LDLT at our center between January 2015 and December 2017 were reviewed retrospectively. During the pretransplant donor evaluation, hepatosteatosis was detected and biopsied donors were included in the study. Ethics committee approval was not obtained because this study was retrospective. CT scans were evaluated by an experienced radiologist. Body mass index and detailed blood tests of donors were obtained from the records received at the time of application.

CT images were taken of all donors by way of multidetection CT (Somatom Sensation 16; Siemens Medical Systems, Erlangen, Germany). CT was performed first without contrast and then with the angiographic imaging contrast. In CT images without contrast, attenuation values of the liver and spleen were calculated by using the mean Hounsfield units (HU) of regions of interest (ROIs). Three ROIs were selected in the right hepatic lobes of all donor candidates, with each ROI measuring nearly $1 \mathrm{~cm} \times 1 \mathrm{~cm}$.

Generally, hepatosteatosis in routine cases is determined by calculating the difference $(\mathrm{CTL}-\mathrm{S})$ between the hepatic attenuation value (CTL) and the spleen attenuation value (CTS) in CT images without contrast material. If this difference is less than five points, then the patient is accepted as being "suspicious for hepatosteatosis."

When the CT images of all donors were reevaluated, the efficacies of two different methods for determining hepatosteatosis were also evaluated and compared with CTL-S. These methods included CTL and the ratio of hepatic attenuation to splenic attenuation (CTL/S).

\section{Liver biopsy procedure}

All hepatic biopsies were performed within one week after hepatosteatosis was confirmed during CT. Prior to the LB, the patient was marked with ultrasonography and then local anesthesia (1\% lidocaine) was applied under the skin. A biopsy needle (Hepafix ${ }^{\circledR}$; B. Braun, Melsungen, Germany) was taken from the intercostal space and biopsied by aspiration technique. Each patient was hospitalized for at least six hours after the procedure. The patients who were admitted to our clinic were followed for at least 24 hours after the procedure. At the end of these respective periods, patients who showed no complications were discharged.

\section{Histopathologic evaluation}

Biopsy materials were fixed in $10 \%$ formalin and embedded in paraffin. The cross-sections were stained with hematoxylin and eosin, Masson's trichrome, and reticulin dyes. Samples were accepted as sufficient for examination if they were larger than $1 \mathrm{~cm}$ in length and contained more than 10 portal areas. Macrovesicular hepatosteatosis was graded according to the percentage and divided into three groups as follows: grade $0(0 \%-5 \%)$, grade $1(6 \%-20 \%)$, or grade $2(>20 \%)[15,16]$. Fibrosis was examined with the use of Masson's trichrome stain.

\section{Results}

The records of the patients who underwent LDLT in our center between January 2015 and December 2017 were retrospectively reviewed. Sixty liver donors (age: 32.4 \pm 7.7 years; 42 males) who underwent LB with hepatosteatosis were included in this study.

According to the results of LB, $43(71.7 \%)$ of 60 patients had grade $0,15(25 \%)$ had grade 1 , and two $(3.3 \%)$ had grade
2 hepatosteatosis, respectively. Since the number of grade 2 patients was low, this group was combined with grade 1 patients for the purpose of statistical computations. Thus, the study participants were ultimately divided into group A (group 0) and group B (groups 1 and 2).

Table 1 presents the demographic data and $\mathrm{CT}$ indices of group A and group B patients.

Table 1 Demographics and CT indexes of donors according to hepatosteatosis

\begin{tabular}{llll}
\hline & Group A (n:43) & Group B (n:17) & P value \\
\hline Age & $32 \pm 7.9$ & $33.4 \pm 7.5$ & $\mathrm{p}>0.05$ \\
Male & $25(58.1 \%)$ & $17(100 \%)$ & $\mathrm{p}<0.05$ \\
AST & $20.5 \pm 6$ & $25.5 \pm 6.9$ & $\mathrm{P}<0.05$ \\
ALT & $22.7 \pm 16.8$ & $32.9 \pm 17.6$ & $\mathrm{P}<0.05$ \\
ALP & $72.3 \pm 20.7$ & $68.4 \pm 14.6$ & $\mathrm{p}>0.05$ \\
GGT & $21.2 \pm 13.5$ & $44.5 \pm 39.1$ & $\mathrm{P}<0.05$ \\
Total Cholesterol & $179.6 \pm 30.5$ & $187.3 \pm 23$ & $\mathrm{P}>0.05$ \\
Triglycerides & $121.7 \pm 64.7$ & $126.7 \pm 60$ & $\mathrm{p}>0.05$ \\
BMI & $26 \pm 3.8$ & $26.8 \pm 3$ & $\mathrm{p}>0.05$ \\
HOMA- IR & $3 \pm 2.3$ & $4.1 \pm 4$ & $\mathrm{P}>0.05$ \\
CTL & $55.5 \pm 7.8$ & $47.6 \pm 6.1$ & $\mathrm{P}<0.05$ \\
CTS & $45.8 \pm 3.4$ & $45.4 \pm 2.7$ & $\mathrm{P}>0.05$ \\
\hline CTL-S & $9.7 \pm 8.6$ & $2.2 \pm 7$ & $\mathrm{p}<0.05$ \\
\hline CTL/S & $1.22 \pm 0.2$ & $1 \pm 0.15$ & $\mathrm{P}<0.05$ \\
\hline
\end{tabular}

AST, aspartate aminotransferase; ALT, alanine aminotransferase; ALP, alkaline phosphatase; GGT, gamma glutamyltransferase; BMI, body mass index; HOMA-IR: Homeostatic model assessment - insulin resistance CTL, liver attenuation; CTL_S, difference between liver and spleen attenuation; $\mathrm{CTL} / \mathrm{S}$, ratio of hepatic attenuation to splenic attenuation; CTS, splenic attenuation.

Negative correlations between the CT indexes [i.e., CTL (r: $-603 ; \mathrm{p}<0.05), \mathrm{CTL}-\mathrm{S}(\mathrm{r}:-578 ; \mathrm{p}<0.05), \mathrm{CTL} / \mathrm{S}$ (r: -568 ; $\mathrm{p}<0.05)]$ and hepatosteatosis were detected.

Receiver operating characteristic analysis was performed (Figure 1) and the three indexes were found to be effective in the diagnosis of hepatosteatosis $(\mathrm{p}<0.001)$. CTL, CTL/S, and CTL-S cutoff values were 48.3, 1.06, and 3.2, respectively; sensitivity and specificity results were $88.3 \%, 64.7 \%$ and $86 \%$, and $64.7 \%$ and $86 \%$; and the area under the curve values were $0.81,0.79$, and 0.80 , respectively.

Figure 1 - The relationship between used computerized tomography (CT) indexes and degree of hepatosteatosis on linear regression analysis.

CTL, hepatic attenuation value; CTL_S, difference between hepatic attenuation and spleen attenuation; $\mathrm{CTL} / \mathrm{S}$, ratio of hepatic attenuation to splenic attenuation.

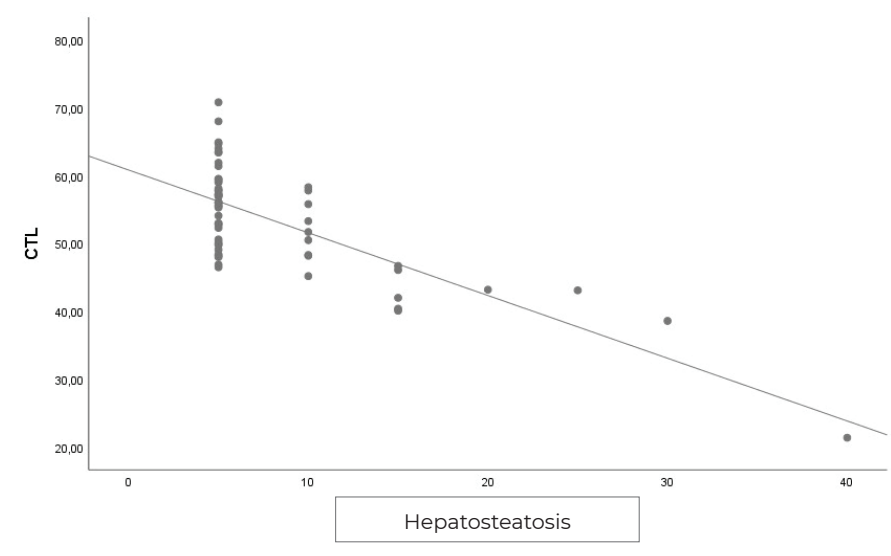



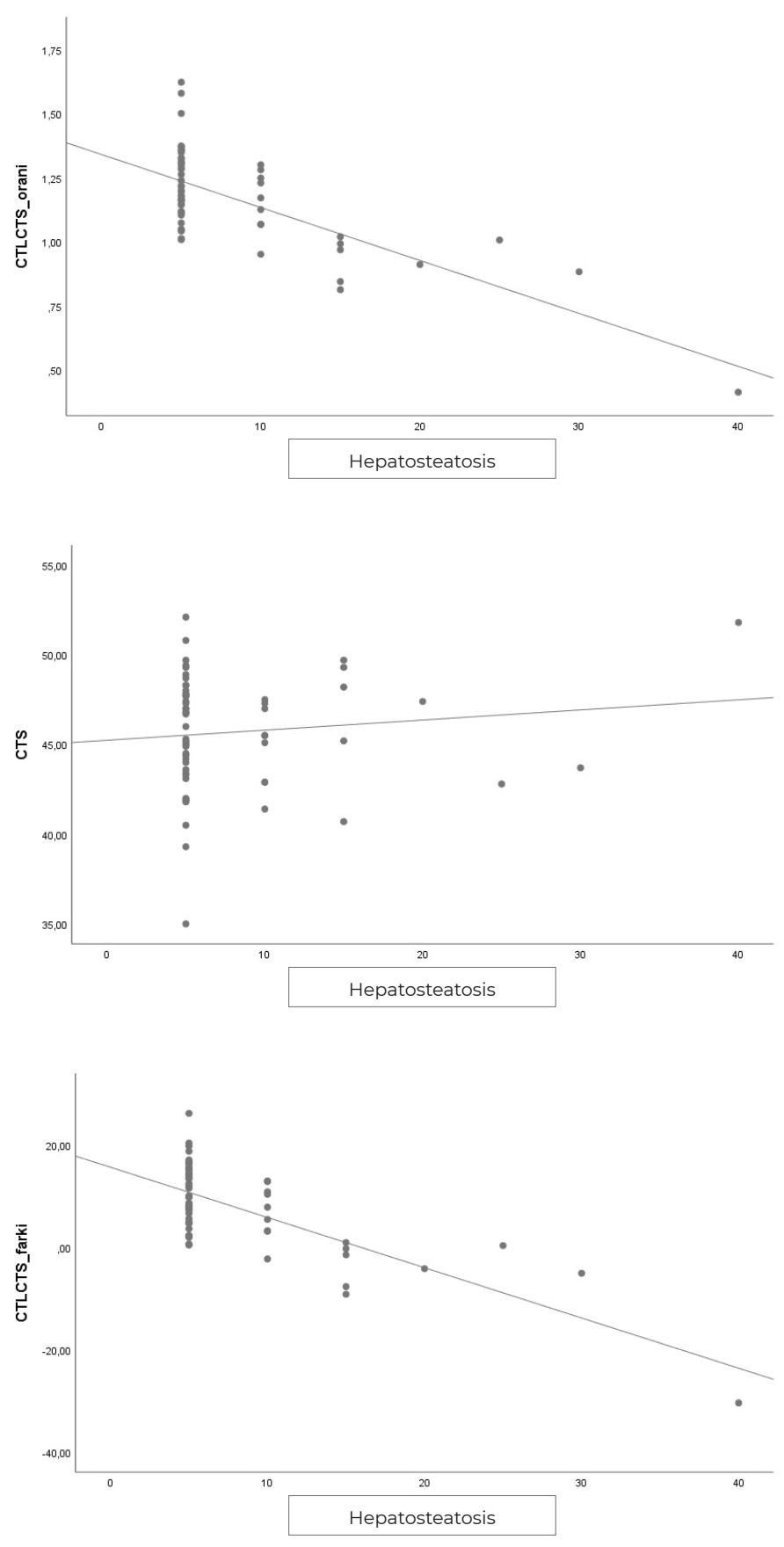

\section{Discussion}

LB was first performed by Erlich in 1883. Currently, it remains the gold-standard method for the diagnosis and followup of most liver diseases. Since it is an invasive procedure, the complications observed during and after the procedure may be serious enough to threaten the life of the involved patients. The biopsy technique, the experience of the applied center, and the suitability of the biopsy needle used are main factors dictating the onset of complications. Today, LB is performed in three ways, as follows: percutaneous, transvenous, and laparoscopic. In our study, percutaneous LB was performed with guidance from ultrasonography. Since bleeding is the most significant cause of mortality, the bleeding parameters of the patient should be closely monitored both before and after the biopsy. Patients with a high risk of bleeding should be treated with blood products against the possibility of bleeding. LB should not be performed in inappropriate patients. Liver transplantation is the most effective treatment of liver cirrhosis. Mostly, LDLT is performed at our center. CT is usually used in donor evaluation. LB is recommended in selected cases where radiological imaging methods cannot obtain sufficient data. Macrovesicular steatosis is associated with complications that may occur postoperatively in LDLT. For these reasons, we are frequently referring to LB in hepatosteatosis in selected cases before LT. One hundred fiftyfive LBs were performed in our center and included in this study and no instances of major bleeding or death due to LB were observed [5-9].

In the current medical literature, many methods have been suggested as alternatives to LB, with CT being the most prominent option among them and the preferred method in our center. The evaluation of hepatosteatosis is performed according to the liver parenchyma attenuation value as measured by HUs. Since the attenuation value of the fat tissue is lower than that of the soft tissue (i.e., $-100 \mathrm{HUs}$ ), the attenuation value of liver parenchyma decreases in the presence of hepatosteatosis.

There are a limited number of studies to date that have compared contrast and noncontrast CT in the evaluation of hepatosteatosis in the literature. Furthermore, depending on the scan timing, there can be value variations in attenuation. For this reason, in the evaluation of hepatosteatosis, $\mathrm{CT}$ without contrast is generally used. In noncontrast CT examinations, the hepatic attenuation value is 55 to $60 \mathrm{HUs}$, which is slightly elevated as compared with that for the spleen due to the glycogen concentration present in the liver. The most frequently used indexes in the evaluation of hepatosteatosis are CTL, CTL/S, and CTL $-\mathrm{S}$. If the CTL and CTS values are low, this indicates hepatosteatosis [10,11]. Park et al. reported the CTL/S sensitivity and specificity as $91 \%$ and $97 \%$, respectively, by establishing the cutoff value of CTL/S as 0.9 in serious hepatosteatosis. In the same study, the authors reported the CTL/S sensitivity and specificity as $91 \%$ and $99 \%$, respectively, by taking a cutoff value of CTL/S of 0.7 [12]. In another study, when the cutoff value was set as high as 1.1 , the sensitivity and the specificity were determined to be $83 \%$ and $82 \%$ [13]. Lee et al. determined, a sensitivity of $72.7 \%$ and a specificity of $91.3 \%$ when CTL $-\mathrm{S}$ cutoff value was 3.2 [14]. In our study, the CTL, CTL/S, and CTL $-\mathrm{S}$ cutoff values were $48.3,1.06$, and 3.2 , respectively. Furthermore, according to these cutoff values, the sensitivity values were $64.7 \%, 64.7 \%$, and $88.3 \%$ and the specificity values were $86 \%, 86 \%$, and $64.7 \%$, respectively, while AUC values for these cutoff levels were found to be $0.81,0.79$, and 0.80 , respectively. All three indices were found to be effective in the diagnosis of hepatosteatosis $(\mathrm{p}<0.001)$.

Importantly, the retrospective nature of this study is a major limitation. Some parameters of the patients could not be collected from the records. However, this study is expected to contribute positively to the medical literature because of its inclusion of a sufficient number of patients in the evaluation of liver donors.

LB continues to be the gold standard in the diagnosis and the treatment of most liver diseases. CT images are widely used and highly correlated with LB findings. As a noninvasive method, CT imaging may decrease the need for LB, which is an invasive procedure, and may reduce the number of complications and the cost of the procedure. Further prospective, multicenter studies are needed to support this hypothesis.

\section{Compliance with ethical standards}

The study conformed to the ethical guidelines of the 1975 Declaration of Helsinki. This retrospective study is verbally consulted to the local ethics committee. The committee secretary verbally declared that ethics approval is not necessary for this retrospective study. For this reason we do not have any certificate of ethics committee. 
Disclosures: For all of authors of this manuscript, there is no potential, personal, financial arrangement (e.g., employment, consultancies, honoraria, stock ownership or options, expert testimony, grants/patents received, and royalties) with a company whose product figures prominently in the submitted manuscript or with a company that makes a competing product.

Acknowledgements: none

Funding: none

\section{References}

1. Tapper EB, Lok AS. Use of Liver Imaging and Biopsy in Clinical Practice. N Engl J Med. 2017; 377(8):756-768. https://doi. org/10.1056/NEJMra1610570.

2. Boyum JH, Atwell TD, Schmit GD, Poterucha JJ, Schleck CD, Harmsen WS, Kamath PS. Incidence and Risk Factors for Adverse Events Related to Image-Guided Liver Biopsy. Mayo Clin Proc. 2016; 91(3):329-35. https://doi.org/10.1016/j. mayocp.2015.11.015. Epub 2016 Feb 2.

3. Braun HJ, Dodge JL, Roll GR, Freise CE, Ascher NL, Roberts JP. Impact of Graft Selection on Donor and Recipient Outcomes After Living Donor Liver Transplantation. Transplantation. 2016; 100(6):1244-50. https://doi.org/10.1097/TP.0000000000001101.

4. Iida T, Masuda K, Matsuyama T, Harada S, Nakamura T, Koshino K, et al. Significance of Proper Graft Selection in Adult LivingDonor Liver Transplant Recipients with Preoperative Deteriorated Condition. Ann Transplant. 2017; 22:541-549. https:/doi. org/10.12659/AOT.904575

5. Angele MK, Rentsch M, Hartl WH, et al. Effect of graft steatosis on liver function and organ survival after liver transplantation. Am J Surg. 2008; 195:214e20. https://doi.org/10.1016/j.amjsurg.2007.02.023

6. Moosburner S, Sauer IM, Gassner JMGV, Schleicher C, Bösebeck D, Rahmel A, et al. Macrosteatosis is a huge problem in liver transplantation-however, not the only one we face. Am J Transplant. 2019. https://doi.org/10.1111/ajt.15418.

7. Núñez K, Thevenot P, Alfadhli A, Cohen A. Complement Activation in Liver Transplantation: Role of Donor Macrosteatosis and Implications in Delayed Graft Function. Int J Mol Sci. 2018; 19(6)pii: E1750. https://doi.org/10.3390/ijms19061750.

8. Maruzzelli L, Parr AJ, Miraglia R, et al. Quantification of hepatic steatosis: a comparison of compterized tomography and magnetic resonance indices in candidates for living liver donation. Acad Radiol. 2014; 21:507e13. https://doi.org/10.1016/j. acra.2014.01.007

9. Limanond P, Raman SS, Lassman C, et al. Macrovesicular hepatic steatosis in living related liver donors: correlation between CT and histologic findings. Radiology. 2004; 230:276e80. https://doi.org/10.1148/radiol.2301021176

10. Lawrence DA, Oliva IB, Israel GM. Detection of hepatic steatosis on contrast-enhanced CT images: diagnostic accuracy of identification of areas of presumed focal fatty sparing. AJR Am J Roentgenol. 2012; 199:44-47. https://doi.org/10.2214/ AJR.11.7838

11. Pickhardt PJ, Park SH, Hahn L, Lee SG, Bae KT, Yu ES. Specificity of unenhanced CT for non invasive diagnosis of hepatic steatosis: implications for the investigation of the natural history of incidental steatosis. Eur Radiol. 2012; 22:1075-1082. https:// doi.org/10.1007/s00330-011-2349-2

12. Park SH, Kim PN, Kim KW, Lee SW, et al. Macrovesicular hepatic steatosis in living liver donors: use of CT for quantitative and qualitative assessment. Radiology. 2006; 239:105e12. https://doi.org/10.1148/radiol.2391050361

13. Iwasaki M, Takada Y, Hayashi M, et al. Noninvasive evaluation of graft steatosis in living donor liver transplantation. Transplantation. 2004; 27(78):1501e5. https://doi.org/10.1097/01.TP.0000140499.23683.0D

14. Lee SS, Park SH, Kim HJ, Kim SY, Kim MY, Kim DY, Suh DJ, Kim KM, Bae MH, Lee JY. Non-invasive assessment of hepatic steatosis: prospective comparison of the accuracy of imaging examinations. J Hepatol. 2010; 52:579-585. https://doi. org/10.1016/j.jhep.2010.01.008 